\title{
Evaluación de ansiedad y frecuencia cardíaca: ensayo clínico aleatorizado de la remoción químico-mecánica de caries con papacárieDuo ${ }^{\mathrm{Tm}}$, proyecto piloto
}

\author{
Elisa-Lopes-Craveiro ${ }^{1}$ \\ Lara-Jansiski-Motta ${ }^{2}$ \\ Thays-Almeida-Alfaya ${ }^{3}$ \\ Camila Haddad-Leal de-Godoy ${ }^{4}$ \\ Laura-Hermida-Bruno ${ }^{5}$ \\ Sandra-Kalil-Bussadori ${ }^{6}$
}

\section{Resumen}

Objetivo: Evaluar el grado de ansiedad y frecuencia cardíaca en pacientes infantiles sometidos a tratamiento con PapacárieDuoTM, comparado a un grupo control con el uso del método tradicional (fresa). Métodos: Se desarrolló un ensayo clínico aleatorizado en 7 pacientes infantiles (5 a 11 años). Fueron tratados 2 molares deciduos en cada niño, realizando la aleatorización para los dos grupos (G1= remoción químico-mecánica de la caries con Papacárie DuoTM; G2= remoción de la dentina cariada utilizando fresa con baja velocidad), realizando un procedimiento restaurador similar en ambos (cemento de ionómero de vidrio) y control. Se evaluó el tiempo de duración del procedimiento, ansiedad y frecuencia cardíaca. Los datos fueron tabulados con el programa SPSS para Windows (v.17) y analizados estadísticamente mediante el
Test Wilcoxon, para los resultados del test VPT; el Test $t$ pareado, para la frecuencia cardíaca, y el Test $t$ para el tiempo de tratamiento, considerándose el nivel de significancia de $5 \%$. Resultados: La media del tiempo para el G1 fue menor ( $p<0.001)$, así como la frecuencia cardíaca en el mismo grupo luego de finalizado el tratamiento ( $p=0.042)$. No hubo diferencia estadística en las reacciones emocionales antes $(\mathrm{p}=0.317)$, durante ( $\mathrm{p}=0.355)$ y después $(\mathrm{p}=0.564)$ de los procedimientos. Conclusión: El tiempo operatorio y la frecuencia cardíaca fueron menores en los niños tratados con el Papacárie DuoTM al ser comparados con los que fueron tratados con tratamiento convencional. Estos hallazgos demuestran las ventajas de los tratamientos conservadores en dientes deciduos.

Palabras clave: caries dental, papaína, ansiedad, frecuencia cardíaca

\footnotetext{
${ }^{1}$ Odontóloga, SP, Brasil. E-mail: liodonto@gmail.com

${ }^{2}$ Profesora de la Disciplina de Odontopediatría, Universidade Nove de Julho, SP Brasil. E-mail: larajmotta@terra.com.br

${ }^{3}$ Especialista en Estomatología. Estudiante del Programa de Maestría en Clínica Odontológica, Universidade Federal Fluminense, RJ, Brasil. E-mail: thalfaya@gmail.com

${ }^{4}$ Especialista en Odontopediatría. Estudiante del Programa de Maestría en Ciencias de Rehabilitación, Universidade Nove de Julho, SP, Brasil. Email: cami_godoy@hotmail.com

${ }^{5}$ Especialista en Docencia Universitaria- U El Bosque, Colombia. Especialista en Odontopediatría- UDELAR, Uruguay. Profesora Unidad de Investigación, Facultad de Odontología, Universidad Católica del Uruguay. Email: laurahermidabruno@gmail.com

${ }^{6}$ Post doctorado en Ciencias. Programa de Maestría en Ciencias de Rehabilitación de la Universidad Nove de Julho y Disciplina de Clínica Integrada de la Universidad Metropolitana de Santos.E-mail: sandra.skb@gmail.com
} 
Artigo Original

\section{Avaliação da ansiedade e freqüência cardíaca: ensaio clínico randomizado da remoção químico-mecânica de cárie com papacárieDuo $^{\mathrm{Tm}}$. projeto piloto}

\section{Resumo}

Objetivo: Avaliar o grau de ansiedade e frequência cardíaca em pacientes infantis submetidos a tratamento com PapacárieDuoTM, comparado a um grupo controle com o uso do método tradicional (broca). Métodos: Desenvolveu-se um ensaio clínico randomizado incluindo 7 pacientes infantis (5 a 11 anos). Foram tratados 2 molares decíduos em cada criança, randomizados para os dois grupos (G1 = remoção químico-mecânica da cárie com Papacárie DuoTM; G2= remoção da dentina cariada com brocas de baixa rotação), sendo realizado procedimento restaurador similar em ambos os grupos (cimento de ionômero de vidro) e controle. Avaliou-se o tempo de duração do procedimento, ansiedade e frequência cardíaca. Os dados foram tabulados com o programa SPSS para Windows (v.17) e analisados estatisticamente mediante o teste Wilcoxon, para os resultados do teste VPT; $o$ teste $t$ pareado para a frequência cardíaca, e o teste $\mathrm{t}$ para o tempo de tratamento, considerandose o nível de significância de 5\%. Resultados: A média do tempo para o G1 foi menor $(p<0.001)$, bem como a frequência cardíaca no mesmo grupo depois de finalizado o tratamento $(\mathrm{p}=0.042)$. Não teve diferença estatística nas reações emocionais antes $(\mathrm{p}=0.317)$, durante $(\mathrm{p}=0.355)$ e depois $(\mathrm{p}=0.564)$ dos procedimentos. Conclusão: $\mathrm{O}$ tempo operatório e a frequência cardíaca foram menores nas crianças tratadas com o Papacárie DuoTM quando comparadas com aquelas que receberam tratamento convencional. Estes achados demonstram as vantagens dos tratamentos conservadores em dentes decíduos.

Palavras-chave: cárie dentária, papaína, ansiedade, frequência cardíaca.

\section{Original article}

\section{Assessment of anxiety and heart rate: randomized clinical trial of chemo-mechanical removal of caries with PapacárieDuo ${ }^{\mathrm{TM}}$. pilot study}

\begin{abstract}
Objective: The aim of the present study was to assess the degree of anxiety and heart rate in pediatric patients submitted to chemomechanical caries removal with Papacárie DuoTM compared to the use of the traditional method
\end{abstract}

(bur and drill). Methods: A randomized clinical trial was carried out involving seven pediatric patients. Two deciduous molars were treated in each child. The teeth were randomly allocated to one of two groups: G1 (chemomechanical caries removal using Papacárie DuoTM and G2 (removal of carious dentin using a bur and low- 
speed drill). The restorative procedure (glass ionomer cement) and follow up were similar in both groups. The time required for the procedure, degree of anxiety and heart rate were determined. The data were analyzed using the SPSS Program for Windows. The Wilcoxon Test was used for the comparison to the VPT results. The paired t-test was used for the comparison of heart rate and the unpaired t-test was used for the time required for treatment. Level of significance was set at 5\% ( $<<0.05)$. Results: Mean time required for the procedure and heart rate at the end of the procedure were lower in G1 $(\mathrm{p}<0.001$ and $\mathrm{p}=0.042$, respectively). No significant differences between groups were found regarding emotional reactions before $(\mathrm{p}=0.317)$, during $(p=0.355)$ or after $(p=0.564)$ the procedures. Conclusion: Operating time and heart rate were lower among the children treated with Papacarie DuoTM in comparison to those submitted to conventional caries removal. These findings demonstrate the advantages of conservative treatment for deciduous teeth.

Key-words: dental caries, papain, anxiety, heart rate.

\section{Introducción}

La caries dental es un problema de salud pública que afecta cerca del 60 a $90 \%$ de niños en países industrializados ${ }^{1}$. En Brasil, el número de niños sin caries aumentó a $44 \%$ luego de la implementación del programa Brasil Sorridente en 2003, que pasó a ofrecer prevención, tratamiento especializado y rehabilitación en todo el país ${ }^{2}$.

El tratamiento de la enfermedad caries dental depende de muchos factores, entre ellos la extensión de las lesiones, pudiendo variar de terapias con el objetivo de remineralizar ${ }^{3}$ las preparaciones cavitarias ${ }^{4}$. Muchas de las tera- pias propuestas provocan miedo y ansiedad en los pacientes ${ }^{5,6}$. Así, investigaciones actuales en odontología han demostrado interés en la búsqueda de instrumentos y técnicas mínimamente invasivas, que logren la preservación de los tejidos dentales y confort del paciente $e^{7-11}$.

La remoción químico mecánica del tejido cariado (CMCR) se basa en el principio de la disolución de la dentina cariada, a través de la aplicación de un agente natural o sintético, facilitando la eliminación del tejido contaminado con auxilio de fuerza mecánica atraumática ${ }^{12}$ y es una alternativa basada en los conceptos de la odontología mínimamente invasiva ${ }^{8}$. El PapacárieTM, producto a base de papaína y cloramina, fue introducido en Brasil en 2003. Este gel promueve la remoción del tejido cariado, preserva el tejido sano y elimina la necesidad de anestesia local8. Estudios clínicos han demostrado resultados satisfactorios con su utilización ${ }^{13-16}$ y en 2011 fue lanzada la nueva versión del producto con la misma efectividad y con algunas propiedades adicionales, denominado Papacárie Duo®TM. Dentro de estas propiedades podemos citar: mayor viscosidad, que permite la utilización del producto sin desperdicio; sin necesidad de refrigeración y validez de dos años.

Dentro de las terapias mínimamente invasivas, es posible que el paciente presente menor incomodidad al recibir tratamiento con instrumentos manuales que con fresas, cuando los anestésicos locales no son utilizados. En un estudio previo fue demostrado que pacientes que recibieron tratamiento restaurador atraumático tuvieron tendencia a presentar menor frecuencia cardíaca $\mathrm{y}$ ansiedad al ser comparados con individuos a los cuales se les había realizado remoción de caries con el uso de instrumentos rotatorios ${ }^{17}$. Basándose en la nueva concepción del producto, el objetivo fue evaluar la ansiedad y miedo, y 
frecuencia cardíaca por medio de un ensayo clínico randomizando comparando dos técnicas, la remoción químico mecánica de caries con el PapacárieDuo®TM y el método tradicional con fresas de baja rotación.

\section{Métodos}

Se desarrolló un ensayo clínico aleatorizado para evaluar el grado de ansiedad y la frecuencia cardíaca en pacientes infantiles sometidos a tratamiento con PapacárieDuo®, comparado a un grupo control con el uso del método tradicional (fresa), con diseño "split mouth" (boca dividida). Todos los responsables recibieron informaciones sobre la investigación y firmaron el consentimiento informado, de acuerdo con la resolución 196/96 del Consejo Nacional de Salud. Este estudio fue aprobado por el Comité de Ética en Investigación Humana, con el n n 045/11.

Fueron selecionados niños entre 5 y 11 años, de ambos sexos, sin distinción de raza, que iban a iniciar tratamiento odontológico en la Clínica Integrada de la Universidad Metropolitana de Santos, Santos, San Pablo. Los criterios de inclusión fueron niños saludables sin alteración sistémica, buen comportamiento, presentar clínicamente por lo menos dos molares deciduos con lesión de caries activa y aguda en dentina, que no sobrepasara 2/3 e involucrara apenas la cara oclusal, cuyo tratamiento permitiera trabajar con visión directa, sin signos ni síntomas clínicos de compromiso pulpar; los criterios de exclusión contemplaron: lesión de caries clase II, III o IV de Black, lesión de caries involucrando solo esmalte, restauraciones deficientes, lesiones cariosas pequeñas en dentina ( $\sin$ acceso para excavadores manuales), lesiones de caries oculta, signos y/o síntomas clínicos de compromiso pulpar e imposibilidad clínica de restauración.
La muestra estuvo compuesta por 7 niños en los que fueron tratados 14 dientes decíduos (primeros y segundos molares), 7 en el grupo experimental y 7 en el grupo control. La aleatorización se realizo mediante sorteo utilizando la piedra lotérica, compuesta por dos números (1 y 2). Los indivíduos sorteados con el número 1, fueron sometidos a remoción químico-mecánica de la caries con Papacárie Duo®TM (Grupo 1 - G1) y los del número 2, al método tradicional, remoción de la dentina cariada utilizando fresa con baja velocidad (Grupo 2 - G2). Todas las etapas de los procedimentos fueron realizadas por un único profesional calibrado.

\section{Intervenciones}

El procedimiento de las intervenciones para recolección de los datos de cada uno de los grupos (experimental y control) siguió el siguiente protocolo.

\section{Grupo 1. Remoción químico-mecánica de la caries con Papacárie DuoTM}

Se realizó radiografía inicial periapical e interproximal y profilaxis con cepillo de Robinson y crema dental fluorada, seguida de aislamiento relativo (retractor de labios, rollos de algodón y eyector de saliva) y registro de tiempo. Se realizó la aplicación del gel Papacárie DuoTM, esperando de 30 a 40 segundos, remoción de la dentina cariada ablandada por el gel por medio del uso de la porción no cortante de las curetas y fueron realizadas reaplicaciones del gel en caso de que todavía hubiese dentina cariada. La evaluación clínica fue realizada por medio de la inspección de la textura de la dentina remanente con sonda exploradora con la finalidad de verificar la total remoción del tejido cariado. Durante este análisis fueron utilizados el examen visual y de 
dureza del tejido dentinario. El procedimiento restaurador fue realizado con Cemento de Ionómero de Vidrio (Ketac Molar Easy mix - 3m ESPETM). Posteriormente se realizó control clínico y radiográfico, con el objetivo de observar la retención del material en la cavidad, presencia de caries secundaria y densidad del área de la dentina remanente.

\section{Grupo 2. Método tradicional: remoción de la dentina cariada utilizando fresa con baja velocidad}

El protocolo inicial fue el mismo que para G1: radiografia inicial periapical e interproximal, profilaxis con cepillo de Robinson y crema dental fluorada, aislamiento relativo y registro de tiempo. Se inició el procedimiento operatorio con la remoción del tejido cariado con fresas de baja velocidad. La evaluación clínica se realizó por medio de la inspección de la textura de la dentina remanente con sonda exploradora, seguida del mismo procedimiento restaurador que para G1, cemento de ionómero de vidrio, registro de tiempo, control clínico y radiográfico.

El registro del tiempo utilizado para la realización del procedimiento fue medido con cronómetro digital (marca Sanny, modelo Progressivo, Brasil) con resolución de 0,01 s, desde el inicio hasta la terminación de la restauración. El tiempo medido fue anotado en una tabla específica para análisis. Todas las intervenciones fueron iniciadas sin previa administración de anestesia local, siendo explicado al niño que la anestesia podría ser aplicada en cualquier momento durante la intervención, si fuera necesario. Para evaluar la necesidad de administrar anestesia local durante los procedimientos, fue utilizado un panel con un diagrama aumentado de la "escala de evaluación facial"18, 19, utilizada para evaluar el dolor. Esa escala es registrada como: 1- sin dolor; 2- leve; 3- moderado; 4- un poco peor; 5 - muy mal; 6 - el peor dolor. Al verificar La presencia de dolor ( 3 a 6) siguiendo la escala, fueron adoptados otros procedimientos como, evaluación de la conducta del profesional, comportamiento del paciente y causa del dolor, para finalmente evaluar la real necesidad de aplicación de anestesia.

Las reacciones emocionales de los niños fueron evaluadas antes, durante y después del tratamiento odontológico utilizando el Venham Picture Test (VPT). La escala fue aplicada por el mismo profesional que realizó los procedimientos. El VPT caracteriza las siguientes reacciones emocionales: neutro (poca ansiedad), alegre (ausencia de ansiedad), miedo (presencia de ansiedad), aflicción-llanto (presencia de ansiedad), triste (presencia de ansiedad), rabia (presencia de ansiedad) y pánico (presencia de ansiedad):

- Las mediciones de la frecuencia cardíaca (FC) fueron realizadas antes, durante, y al final de la atención odontológica por medio de la utilización de frecuencímetro (marca Choicemmed, modelo Oxywatch C20 Pulse Oximeter, China).

- Los datos fueron tabulados con el programa SPSS para Windows (v.17) y analizados estadísticamente mediante el Test Wilcoxon, para los resultados del test VPT; el Test t pareado, para la frecuencia cardíaca, y finalmente el Test $t$ para el tiempo de tratamiento, considerándose el nivel de significancia de $5 \%$.

\section{Resultados}

El tiempo empleado en ambas técnicas puede ser observado en la tabla 1. La media de tiempo para G1 fue relativamente menor $(\mathrm{p}<0.001)$. 
Solo un paciente de G2 relató dolor durante el procedimiento. El dolor fue clasificado como leve y no fue registrada la necesidad de anestesia local.

Los niños pertencientes al G1 presentaron reacciones emocionales más positivas durante el tratamiento, siendo la respuesta "Alegre", relacionada ausencia de ansiedad escogida por $42,9 \%$ de los niños, mientras que en G2 "Miedo" fue indicada por el mismo número de niños, aunque la diferencia entre los grupos no fue estadísticamente significativa (Tabla 2).
La frecuencia cardíaca tanto durante como después de la realización del procedimiento fue menor en G1. En el análisis entre las técnicas, hubo significancia estadística de la FC después de la finalización del tratamiento $(\mathrm{p}=0.042)$ (Tabla 3).

\section{Discusión}

Los resultados de este estudio demuestran que el tiempo utilizado en el procedimiento con el PapacárieDuoTM es menor comparado con el método tradicional. No hubo asociación entre

Tabla 1. Tiempo de realización del procedimiento en minutos de acuerdo con la técnica empleada (Test T).

\begin{tabular}{|c|c|c|c|c|c|c|}
\hline & Média & Desvío padrón & $\mathbf{T}$ & Valor de $p$ & \multicolumn{2}{|c|}{ Intervalo de Confianza $95 \%$} \\
\hline PapacárieDuoTM & 22,752 & 1,94616 & 30,932 & \multirow{2}{*}{$\mathrm{p}<0,001^{*}$} & 20,9530 & 24,5528 \\
\hline Método tradicional & 26,138 & 1,96759 & 35,148 & & 24,3189 & 27,9583 \\
\hline
\end{tabular}

Tabla 2. Análisis de las reacciones emocionales por medio del VPT, comparación entre los dos procedimientos (Test Wilcoxon).

\begin{tabular}{|c|c|c|c|c|c|c|c|c|c|}
\hline & \multicolumn{3}{|c|}{ Antes } & \multicolumn{3}{|c|}{ Durante } & \multicolumn{3}{|c|}{ Después } \\
\hline & G1 & G2 & P-valor & G1 & G2 & P-valor & G1 & G2 & P-valor \\
\hline Neutro & $\begin{array}{c}6 \\
(85,7 \%) \\
\end{array}$ & $\begin{array}{c}7 \\
(100 \%)\end{array}$ & \multirow{7}{*}{0,317} & $\begin{array}{c}4 \\
(57,1 \%) \\
\end{array}$ & $\begin{array}{c}4 \\
(57,1 \%)\end{array}$ & \multirow{7}{*}{0,335} & $\begin{array}{c}5 \\
(71,4 \%) \\
\end{array}$ & $\begin{array}{c}6 \\
(85,7 \%) \\
\end{array}$ & \multirow{7}{*}{0,564} \\
\hline Alegre & $\begin{array}{c}1 \\
(14,3 \%)\end{array}$ & - & & $\begin{array}{c}3 \\
(42,9 \%)\end{array}$ & - & & $\begin{array}{c}2 \\
(28,6 \%)\end{array}$ & $\begin{array}{c}1 \\
(14,3 \%)\end{array}$ & \\
\hline Miedo & - & - & & - & $\begin{array}{c}3 \\
(42,9 \%)\end{array}$ & & - & - & \\
\hline Afligido/llanto & - & - & & - & - & & - & - & \\
\hline Triste & - & - & & - & - & & - & - & \\
\hline Rabia & - & - & & - & - & & - & - & \\
\hline Pánico & - & - & & - & - & & - & - & \\
\hline
\end{tabular}

Tabla 3. Frequencia cardíaca (pulsaciones por minuto) entre los dos procedimientos (Teste T pareado)

\begin{tabular}{|c|c|c|c|c|c|c|}
\hline \multirow{2}{*}{} & \multicolumn{3}{|c|}{ Durante } & \multicolumn{3}{c|}{ Después } \\
\cline { 2 - 6 } & G1 & G2 & p-valor & G1 & G2 & p-valor \\
\hline Média & 81,8571 & 96,7143 & & 86,2857 & 103,7143 & \multirow{2}{*}{0,042} \\
\hline Desvío padrón & 15,53950 & 3,30224 & 0,101 & 5,64843 & 23,26452 & \\
\hline
\end{tabular}


reacciones emocionales y las técnicas de remoción de caries empleadas, mientras que la FC se presenta significativamente disminuída después de la finalización del tratamiento en pacientes sometidos a CMCR.

La optimización del tiempo en la odontologia es de extrema importancia, principalmente al tratar pacientes infantiles y ansiosos. Los resultados relativos a esa variable corroboran los estudios anteriores que utilizaron la $\mathrm{CMCR}^{20-22}$. Eso puede ser atribuído al hecho de que en la CMCR el objetivo principal es la eliminación del tejido cariado, no habiendo necesidad de preparar las paredes de la cavidad para recibir el material restaurador. La colaboración del paciente es un item a ser considerado en ese caso. Debido a la disminución de los niveles de miedo, los atrasos derivados del disconfort de los pacientes fueron menores, posibilitando una atención más fluída por medio de la utilización del PapacárieDuoTM.

La utilización de instrumentos rotatorios es el método más común de remoción de lesiones de caries, aunque el mismo es acompañado de dolor, disconfort y ansiedad ${ }^{23}$. La menor FC encontrada en el tratamiento con PapacárieDuoTM puede significar que la técnica es menos agresiva y por lo tanto, menos traumática para los pacientes. La menor incomodidad ocurre debido al uso de curetas no cortantes, la acción local de la papaína favoreciendo el proceso de reparación y el $\mathrm{pH}$ neutro del producto $^{13}$. Estudios similares han investigado cuestiones relativas al método tradicional (MT) y los mínimamente invasivos. Van Bochove y Van Amerongen (2006) analizaron las posibles diferencias de los grados de disconfort entre el tratamiento restaurador atraumático (ART) y el MT con y sin analgesia. Fue observado menor incomodidad con ART, siendo éste el preferido por los pacientes ${ }^{24}$. Otro estudio con ART y MT verificó la FC durante todas las fases del procedimiento restaturador (entrance, start, deep excavation, matrix, restoration and end). La FC se mostró significativamente menor durante la deep excavation de la $\operatorname{ART}(\mathrm{p}=0.03)$. En este estudio se resaltó que el sexo, nivel de ansiedad y el profesional desempeñan un papel importante en el comportamiento del niño durante el procedimiento ${ }^{17}$.

La FC no puede ser atribuída a ansiedad. Diversos instrumentos pueden ser útiles en su evaluación ${ }^{25}$, como es el caso del VPT, utilizado en el presente estudio. Algunos cuadros de ansiedad pueden estar presentes durante la atención odontológica. Una investigación analizó la ansiedad de niños en relación a las conductas odontológicas en dos momentos. El primero a los 5 años de edad y el segundo a los 10. Los resultados demostraron que los niños que presentaban más lesiones de caries tendían a ser más ansiosos en el futuro. Esto se debe al condicionamiento clásico, incluyendo el dolor de los procedimentos y otras experiencias negativas durante el tratamiento, como los estímulos incondicionados ${ }^{26}$. A pesar de la ausencia de significancia estadística entre las reacciones emocionales y las técnicas, individuos del G2 presentaron un cuadro de miedo durante el procedimiento. Al analizar estas variables también debe ser tenida en cuenta la franja etaria, pues hay algunas diferencias en el desarrollo emocional del niño. En este estudio, la muestra estaba comprendida entre 5 y 11 años y esto corresponde a dos etapas de desarrollo emocional de Piaget. La etapa que corresponde a las edades de 2 a 7 años es denominada pre-operatoria y está marcada por el dominio del lenguaje, animismo, finalismo y antropocentrismo/ egocentrismo, mientras que la siguiente fase de los 7 a los 11 años es denominada como el período de las operaciones concretas, donde ocurre el inicio de la capacidad de utilizar la lógica. El 
niño es capaz de estabelecer relaciones y contemplar puntos de vista diferentes ${ }^{27}$.Un factor limitante para obtener conclusiones de mayor validez puede haber sido el tamaño reducido de la muestra y la cantidad de variables pertenecientes a las reacciones emocionales. Por lo tanto, se sugiere una muestra mayor para obtener resultados más conclusivos y que los pacientes sean categorizados de acuerdo con las etapas de desarrollo, de modo que la percepción sobre el tratamiento sea la más fiel posible.
En conclusión, los resultados de este estudio demostraron que el PapacárieDuoTM seguido de restauración con cemento de ionómero de vidrio es una opción viable de tratamiento dentro de los conceptos de odontología mínimamente invasiva. Además de eso, el tiempo utilizado y la frecuencia cardíaca posterior al proocedimiento fueron significativamente menores que los del método tradicional, demostrando las ventajas del tratamiento conservador en molares deciduos.

\section{Bibliografía}

1. WHO. World Health Organization. What is the burden of oral disease? Disponible in http://www.who.int/oral_ health/disease_burden/global/en/. Acessed 07/03/2012.

2. Teixeira KIR, Bueno AC, Cortés ME. Processos Físico-Químicos no Biofilme Dentário Relacionados à Produção da Cárie. Química nova na escola. 2010;32(3).

3. Arnold WH, Gaengler P. Light- and electronmicroscopic study of infiltration of resin into initial caries lesions--a new methodological approach. J Microsc. 2012;245(1):26-33.

4. Tveit AB, Espelid I, Skodje F. Restorative treatment decisions on approximal caries in Norway. Int Dent J. 1999;49(3):16572.

5. Bergius M, Berggren U, Bogdanov O, Hakeberg M. Dental anxiety among adolescents in St. Petersburg, Russia. Eur J Oral Sci. 1997;105(2):117-22.

6. Lourenco-Matharu L, Ashley PF, Furness S. Sedation of children undergoing dental treatment. Cochrane Database Syst Rev. 2012;3:CD003877.

7. Balciuniene I, Sabalaite R, Juskiene I. Chemomechanical caries removal for children. Stomatologija. 2005;7(2):40-4.

8. Bussadori SK, Castro LC, Galvao AC. Papain gel: a new chemo-mechanical caries removal agent. J Clin Pediatr Dent. 2005;30(2):115-9.

9. Correa FN, Rocha Rde O, Rodrigues Filho LE, Muench A, Rodrigues CR. Chemical versus conventional caries removal techniques in primary teeth: a microhardness study. J Clin Pediatr Dent. 2007;31(3):187-92.

10. Lopez N, Simpser-Rafalin S, Berthold P. Atraumatic restorative treatment for prevention and treatment of caries in an underserved community. Am J Public Health. 2005;95(8):1338-9.

11. Mickenautsch S, Yengopal V, Banerjee A. Atraumatic restorative treatment versus amalgam restoration longevity: a systematic review. Clin Oral Investig. 2010;14(3):233-40.

12. Mathre S, Kumar S, Sinha S, Ahmed BMN, Thanawala EA. Chemo-Mechanical Method Of Caries Removal: A Brief Review. IJCDS. 2011;2(2):52-7.

13. Bussadori SK, Guedes CC, Bachiega JC, Santis TO, Motta LJ. Clinical and radiographic study of chemical-mechanical removal of caries using Papacarie: 24-month follow up. J Clin Pediatr Dent. 2011;35(3):251-4.

14. Bussadori SK, Guedes CC, Hermida Bruno ML, Ram D. Chemo-mechanical removal of caries in an adolescent patient using a papain gel: case report. J Clin Pediatr Dent. 2008;32(3):177-80.

15. Piva E, Ogliari FA, Moraes RR, Cora F, Henn S, Correr-Sobrinho L. Papain-based gel for biochemical caries removal: influence on microtensile bond strength to dentin. Braz Oral Res. 2008;22(4):364-70. 
16. Kotb RM, Abdella AA, El Kateb MA, Ahmed AM. Clinical evaluation of Papacarie in primary teeth. J Clin Pediatr Dent. 2009;34(2):117-23.

17. Schriks MC, van Amerongen WE. Atraumatic perspectives of ART: psychological and physiological aspects of treatment with and without rotary instruments. Community Dent Oral Epidemiol. 2003;31(1):15-20.

18. Whaley L, Wong DLN. Nursing care of infants and children. 3 ed. St. Louis: Mosby; 1987.

19. Wong DL, Baker CM. Pain in children: comparison of assessment scales. Pediatr Nurs. 1988;14(1):9-17.

20. Jawa D, Singh S, Somani R, Jaidka S, Sirkar K, Jaidka R. Comparative evaluation of the efficacy of chemomechanical caries removal agent (Papacarie) and conventional method of caries removal: an in vitro study. J Indian Soc Pedod Prev Dent. 2010;28(2):73-7.

21. Carrillo CM, Tanaka MH, Cesar MF, Camargo MAF, Juliano Y, Novo NF. Use of Papain Gel in Disabled Patients. JDC. 2008;75(3):222-8.

22. Aguilar AAA, Caro TER, Saavedra JH, França CM, Fernandes KPS, Mesquita-Ferrari RA, et al. La práctica restaurativa atraumática: una alternativa dental bien recibida por los niños. Rev Panam Salud Publica. 2012;31(2):150-2.

23. Nadanovsky P, Cohen Carneiro F, Souza de Mello F. Removal of caries using only hand instruments: a comparison of mechanical and chemo-mechanical methods. Caries Res. 2001;35(5):384-9.

24. Van Bochove JA, van Amerongen WE. The influence of restorative treatment approaches and the use of local analgesia, on the children's discomfort. Eur Arch Paediatr Dent. 2006;7(1):11-6.

25. Guinot Jimeno F, Yuste Bielsa S, Cuadros Ferna Ndez C, Lorente Rodriguez AI, Mercade Bellido M. Objective and subjective measures for assessing anxiety in paediatric dental patients. Eur J Paediatr Dent. 2011;12(4):239-44.

26. Raadal M, Strand GV, Amarante EC, Kvale G. Relationship between caries prevalence at 5 years of age and dental anxiety at 10. Eur J Paediatr Dent. 2002;3(1):22-6.

27. Ferracioli L. Aprendizagem, desenvolvimento e conhecimento na obra de Jean Piaget: uma análise do processo de ensino-aprendizagem em Ciências. R bras Est pedag. 1999;80(194):5-18.

Revisión y traducción de portugués: Fabián Calixto Fraiz

Recibido: 05-02-12

Aceptado: 17-02-13

Correspondencia: thalfaya@gmail.com 